\title{
Coupled Shape Priors for Dynamic Segmentation of Dendritic Spines
}

\author{
Naeimeh Atabakilachini*, Ertunc Erdil*, A. Ozgur Argunsah ${ }^{\dagger}$, Lavdie Rada ${ }^{\ddagger}$, Devrim Unay ${ }^{\S}$, Mujdat Cetin* \\ *Sabanci University, Faculty of Engineering and Natural Sciences, Istanbul, Turkey \\ \{naeimeh, ertuncerdil, mcetin\}@sabanciuniv.edu \\ ${ }^{\dagger}$ Zurich University, Institute of Brain Research, Zurich, Switzerland \\ argunsah@hifo.uzh.ch \\ $\ddagger$ Bahcesehir University, Faculty of Engineering and Natural Sciences, Istanbul, Turkey \\ lavdie.rada@eng.bau.edu.tr \\ §Izmir Economy University, Department of Biomedical Engineering, Izmir, Turkey \\ devrim.unay@ieu.edu.tr
}

\begin{abstract}
Segmentation of biomedical images is a challenging task, especially when there is low quality or missing data. The use of prior information can provide significant assistance for obtaining more accurate results. In this paper we propose a new approach for dendritic spine segmentation from microscopic images over time, which is motivated by incorporating shape information from previous time points to segment a spine in the current time point. In particular, using a training set consisting of spines in two consecutive time points to construct coupled shape priors, and given the segmentation in the previous time point, we can improve the segmentation process of the spine in the current time point. Our approach has been evaluated on 2photon microscopy images of dendritic spines and its effectiveness has been demonstrated by both visual and quantitative results.

Index Terms-Dynamic segmentation, dendritic spine segmentation, 2-photon microscopy, nonparametric shape priors, coupled shape priors.
\end{abstract}

\section{INTRODUCTION}

Dendritic spines are small protrusions covering the surface of a dendrite and have two main parts, spine head and spine neck [1]. Spines are one of the crucial components of neurons and have been widely studied over the last century. It has been observed that spines have spontaneous and rapid changes in their structure [2], [3] and it has also been suggested that morphology and density of spines are highly correlated with their cognitive functionality, such as memory and learning [4], [5], [6]. Considering the importance of deducing the crucial role of spines in those functionalities, analysis of spines has become a major topic of interest in neurobiology. Automating dendritic spine analysis can aid neuroscientists in their studies, since performing manual analysis can be a tedious task and subject to human bias.

Proper analysis of morphological changes of a spine over a time interval requires efficient and accurate processing of time-lapse neuron images, particularly to obtain the spine contour through segmentation. 2-photon microscopy images of a dendrite in consecutive time points is shown in Figure 1. In the case of spines with neck, the segmentation problem is even more challenging, since in 2-photon microscopy images,

978-1-5090-6494-6/17/\$31.00 2017 IEEE
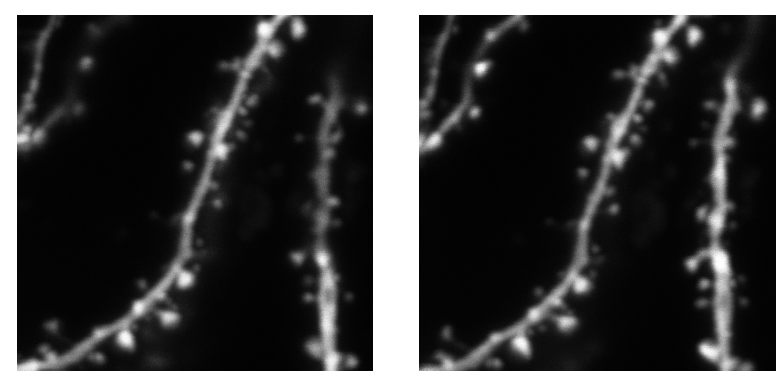

Fig. 1: 2-photon microscopy images of a dendrite in consecutive time points.

the neck does not exhibit strong contrast. Overall, purely datadriven segmentation of spines is challenging and prior information about the shape of the spine leads to more successful segmentation.

Several methods have been proposed for spine segmentation in 2-photon microscopy images in the literature. Thresholdingbased segmentation is performed in [7], [8]. As mentioned before, since the neck part of the spine has low contrast, these methods are not successful in finding the appropriate threshold in such cases.

Shape prior based methods were introduced before for segmentation of deformable objects. A typical active-contour model enforces a curve length penalty on the underlying shapes. This constraint is not sufficient in many applications and more structured prior knowledge is required.

Early shape-based segmentation algorithm employ principal component analysis (PCA) of the signed distance function representation of training samples to characterize shape variability [9]. A nonparametric shape prior model has been proposed by Kim et al. [10] which estimates the underlying shape distribution by extending a Parzen density estimator to the space of shapes. Then, an optimization problem for maximum a posteriori (MAP) estimation is solved by active contours. Erdil et al. [11] present a joint classification and segmentation approach for dendritic spine segmentation which infers the class of the spine during the segmentation process 
and continues the remaining process adaptively. Another work by Erdil et al. [12] proposes nonparametric joint shape and feature priors for segmentation of dendritic spines. They add discriminative features to the density estimation process and incorporate the learned joint shape and feature prior distribution into a maximum a posteriori estimation framework for segmentation.

While two-photon microscopy involves collecting spine images over a time interval, existing techniques are concerned with segmenting spines at a single time point without regard to the data at other time points. In this paper, we view spine segmentation over time series images as a dynamic estimation problem, and propose a preliminary approach with this perspective. Spine shapes evolve over time and information about the spine contour from the previous time points would in principle provide useful prior information for segmentation of the spine at the current time point. To this end, from manually segmented training data, we learn joint (or coupled) nonparametric shape densities of spines in consecutive time points. Then in the process of segmenting a spine at a particular time point, given its segmentation in the previous time point, we use such learned densities to incorporate information from the previous segmentation as prior shape information into the segmentation process.

\section{PROPOSED APPROACH}

In this section, the derivation of the mathematical formulation and a detailed explanation of our proposed approach is provided.

Let us assume that we have $n$ training shapes $\mathbf{C}^{(\mathbf{t}-\mathbf{1})}=$ $\left\{C_{1}^{(t-1)}, C_{2}^{(t-1)}, \ldots, C_{n}^{(t-1)}\right\}$ at time $(t-1)$. Let us also assume that we have $n$ training shapes $\mathbf{C}^{(\mathbf{t})}=$ $\left\{C_{1}^{(t)}, C_{2}^{(t)}, \ldots, C_{n}^{(t)}\right\}$ at time $(t)^{1}$. Note that $C_{i}^{(t-1)}$ and $C_{i}^{(t)}$ are boundaries of the same spine in consecutive time points for each $i \in[1, n]$. Then, the posterior probability of $C^{(t)}$ given intensity images $y^{(t-1)}$ at time point $(t-1)$ and $y^{(t)}$ at time point $(t)$ is written as

$p\left(C^{(t)} \mid y^{(t-1)}, y^{(t)}\right)=\int p\left(C^{(t)}, C^{(t-1)} \mid y^{(t-1)}, y^{(t)}\right) \mathrm{d} C^{(t-1)}$.

Using Bayes' rule and the assumption that $y^{(t-1)}$ and $y^{(t)}$ are independent conditioned on the corresponding spine boundaries,we get

$$
\begin{aligned}
& p\left(C^{(t)} \mid y^{(t-1)}, y^{(t)}\right) \\
& \propto \int p\left(y^{(t-1)}, y^{(t)} \mid C^{(t)}, C^{(t-1)}\right) p\left(C^{(t)}, C^{(t-1)}\right) \mathrm{d} C^{(t-1)} \\
& =\int p\left(y^{(t-1)} \mid C^{(t-1)}\right) p\left(y^{(t)} \mid C^{(t)}\right) p\left(C^{(t)} \mid C^{(t-1)}\right) p\left(C^{(t-1)}\right) \mathrm{d} C^{(t-1)} \\
& \propto p\left(y^{(t)} \mid C^{(t)}\right) \int p\left(C^{(t)} \mid C^{(t-1)}\right) p\left(C^{(t-1)} \mid y^{(t-1)}\right) \mathrm{d} C^{(t-1)}
\end{aligned}
$$

From this point on, one can proceed with various assumptions on the probability densities in Equation (2). In this paper, we learn $p\left(C^{(t)} \mid C^{(t-1)}\right)$ nonparametrically from the training data

\footnotetext{
${ }^{1}$ Note that the shapes in both $C^{(t-1)}$ and $C^{(t)}$ have already been aligned to remove the shape variations due to pose differences
}

and use the data term proposed in [13] for $p\left(y^{(t)} \mid C^{(t)}\right)$ which we explain in detail later in this section. For the posterior density $p\left(C^{(t-1)} \mid y^{(t-1)}\right)$, in this preliminary work we assume that it is a delta function which can be written as follows:

$$
p\left(C^{(t-1)} \mid y^{(t-1)}\right)=\delta\left(C^{(t-1)}-C^{\prime}\right)
$$

where $\delta($.$) is the Dirac delta function and C^{\prime}$ is the perfect segmentation of the spine at time point $(t-1)$ which we further assume that we already have. Then, Equation (2) becomes

$$
p\left(C^{(t)} \mid y^{(t-1)}, y^{(t)}\right) \propto p\left(y^{(t)} \mid C^{(t)}\right) p\left(C^{(t)} \mid C^{\prime}\right) .
$$

Finally, the energy function to be minimized can be written by taking the negative logarithm of Equation (4) as

$$
E\left(C^{(t)}\right)=-\log p\left(y^{(t)} \mid C^{(t)}\right)-\log p\left(C^{(t)} \mid C^{\prime}\right) .
$$

For the first term $-\log p\left(y^{(t)} \mid C^{(t)}\right)$ in Equation (5), we use the piecewise-constant version of the Mumford functional [14] proposed in [13] which is given by:

$$
\begin{aligned}
-\log p\left(y^{(t)} \mid C^{(t)}\right) & =\int_{C_{\text {in }}^{(t)}}\left(I(x)-m_{\text {in }}\right)^{2} \mathrm{~d} x \\
& +\int_{C_{\text {out }}^{(t)}}\left(I(x)-m_{\text {out }}\right)^{2} \mathrm{~d} x
\end{aligned}
$$

where $m_{\text {in }}\left(m_{\text {out }}\right)$ are the mean intensities inside (outside) of curve $C^{(t)}$. We learn the second term $p\left(C^{(t)} \mid C^{\prime}\right)$ in Equation (5) nonparametrically from the training data using Parzen density estimation as follows:

$$
p\left(C^{(t)} \mid C^{\prime}\right) \propto \frac{1}{n} \sum_{i=1}^{n} k\left(d_{L_{2}}\left(C^{(t)}, C_{i}^{(t)}\right), d_{L_{2}}\left(C^{\prime}, C_{i}^{(t-1)}\right), \sigma\right)
$$

where $k(., ., \sigma)$ is a $2 D$ Gaussian with standard deviation $\sigma$ and $d_{L_{2}}(.,$.$) is the L_{2}$ distance metric.

We minimize the energy function in Equation (5) using gradient descent. In order to propagate the curve, we use level set representation of binary shapes which we denote by $\phi$. We use the sign convention $\phi<0$ for inside the curve and $\phi>0$ for outside the curve. The overall gradient flow of the energy function is the sum of the two terms, one based on the data term and the other based on the coupled shape prior term. The partial derivative of the data term is computed as follows:

$\frac{-\partial \log p\left(y^{(t)} \mid \phi^{(t)}\right)}{\partial \phi^{(t)}}=\beta\left[-\left(I(x)-m_{\text {in }}\right)^{2}+\left(I(x)-m_{\text {out }}\right)^{2}\right] \vec{N}$

where $\vec{N}$ is the outward curve normal. Also, the partial derivative of the coupled shape prior term is given by

$$
\begin{aligned}
& \frac{\partial \log p\left(\phi^{(t)} \mid \phi^{\prime}\right)}{\partial \phi^{(t)}}=\frac{1}{p\left(\phi^{(t)} \mid \phi^{\prime}\right)} \frac{1}{\sigma^{2}} \frac{1}{n} \\
& \sum_{i=1}^{n} k\left(d_{L_{2}}\left(\phi^{(t)}, \phi_{i}^{(t)}\right), d_{L_{2}}\left(\phi^{\prime}, \phi_{i}^{(t-1)}\right), \sigma\right)\left(\phi_{i}^{(t)}-\phi^{(t)}\right) .
\end{aligned}
$$

In our approach, we start the segmentation with the level set representation of an initial curve and update the curve with the gradient of the energy function in Equation (5). Finally, when the curve converges at some point, $C^{(t)}$ can be found from $\phi^{(t)}$ by thresholding at zero. 


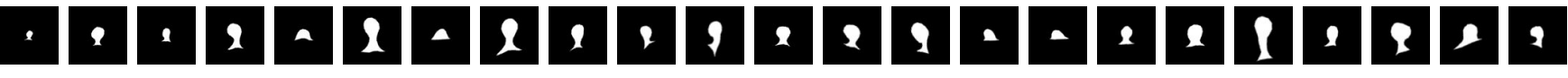

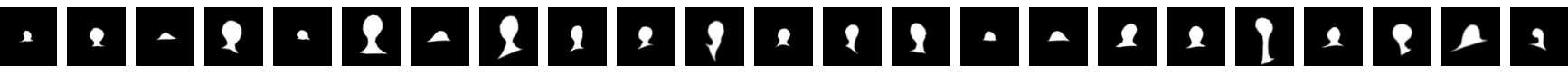

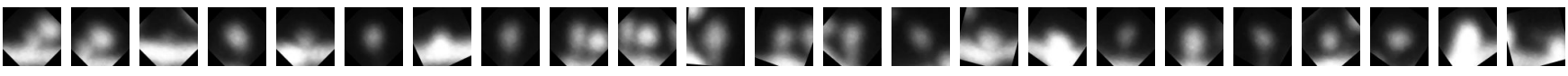

Fig. 2: The dendritic spine data set. First row: manual segmentations in the first time point, second row: manual segmentations in the second time point, third row: intensity images in the second time point.

\section{EXPERIMENTAL RESULTS}

In this section, we present experimental results of our dynamic segmentation approach on the dendritic spine data set. The dendritic spine data set contains 23 spine images in consecutive time points together with their manual segmentations as shown in Figure 2. We perform experiments on a leave-one-out fashion by using an intensity image for test and the remaining 22 binary images in consecutive time points for training.

We compare the performance of the proposed approach with the approach proposed in [10] which does not use any shape priors from the previous time point. We evaluate the performance of the proposed approach and the approach in [10] by comparing the segmentation results with manual segmentations of a domain expert using Dice score [15]. Dice score measures the similarity between two sets $X$ and $Y$ which are in our case binary images representing the ground truth and segmentation results of the segmentation algorithm. Dice score takes values between 0 and 1 where 1 indicates perfect match between $X$ and $Y$. Dice score is computed as follows:

$$
D(X, Y)=\frac{2|X \cap Y|}{|X|+|Y|} .
$$

We provide the quantitative results of both the proposed approach and the approach of Kim et al. [10] in Table I. The quantitative results demonstrate that the proposed approach improves the segmentation results by using the segmentation from the previous time point as prior information.

We also provide some visual results obtained by the proposed approach (see Figure 3) and the approach of Kim et al. [10] (Figure 4). The visual results also demonstrate that the proposed approach improves the segmentation results of the approach proposed in [10].

\section{Conclusion}

In this paper a dendritic spine segmentation method that employs coupled shape priors exploiting information about the boundary from the previous time point is proposed. Our approach is motivated by the assumption that over a time period, there are co-dependencies between changes of spines whose shapes are similar initially. Based on that, a coupled shape prior term has been constructed for providing the shape force in the curve evolution process. The resulting energy function has been minimized using gradient descent and level sets. We evaluate the effectiveness of the proposed method
TABLE I: Dice Score results.

\begin{tabular}{|l|c|c|}
\hline & Proposed Method & Kim et al. [10] \\
\hline Spine 1 & 0.6386 & 0.6379 \\
\hline Spine 2 & 0.7520 & 0.7470 \\
\hline Spine 3 & 0.6343 & 0.6366 \\
\hline Spine 4 & 0.7847 & 0.6727 \\
\hline Spine 5 & 0.6627 & 0.6655 \\
\hline Spine 6 & 0.7937 & 0.6673 \\
\hline Spine 7 & 0.6689 & 0.6906 \\
\hline spine 8 & 0.7905 & 0.6789 \\
\hline Spine 9 & 0.7488 & 0.6414 \\
\hline Spine 10 & 0.7414 & 0.6643 \\
\hline Spine 11 & 0.7536 & 0.6693 \\
\hline Spine 12 & 0.7299 & 0.6435 \\
\hline Spine 13 & 0.7703 & 0.6753 \\
\hline Spine 14 & 0.7523 & 0.5977 \\
\hline Spine 15 & 0.6872 & 0.7057 \\
\hline Spine 16 & 0.6908 & 0.7145 \\
\hline Spine 17 & 0.6965 & 0.5955 \\
\hline Spine 18 & 0.8041 & 0.7352 \\
\hline Spine 19 & 0.8079 & 0.6626 \\
\hline Spine 20 & 0.7230 & 0.6473 \\
\hline Spine 21 & 0.7963 & 0.6566 \\
\hline Spine 22 & 0.7903 & 0.7696 \\
\hline Spine 23 & 0.7162 & 0.6700 \\
\hline Average & $\mathbf{0 . 7 3 6 2}$ & $\mathbf{0 . 6 7 1 5}$ \\
\hline
\end{tabular}
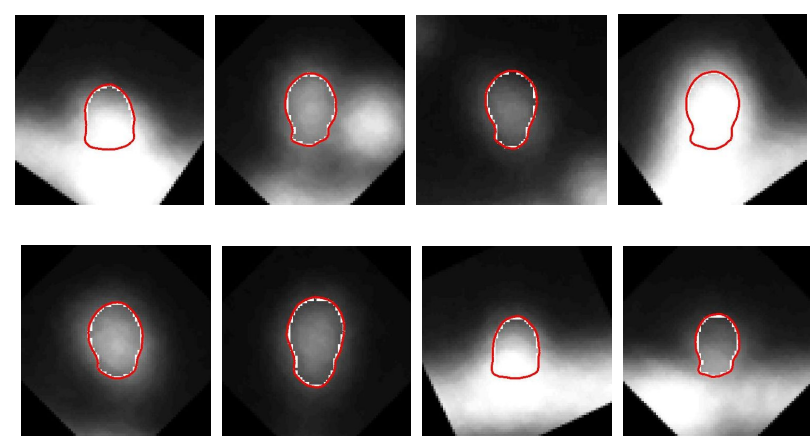

Fig. 3: Visual segmentation results obtained by the proposed method.

on 2-photon microscopy images of spines. According to both visual and quantitative experimental results, our method is more successful in the segmentation process of the spine in comparison with Kim et al. [10], which uses static shape priors rather than dynamic priors based on the previous time point.

A possible future approach would be to extend this preliminary study to a complete dynamic estimation setting which uses imperfect segmentations from previous time points. This 

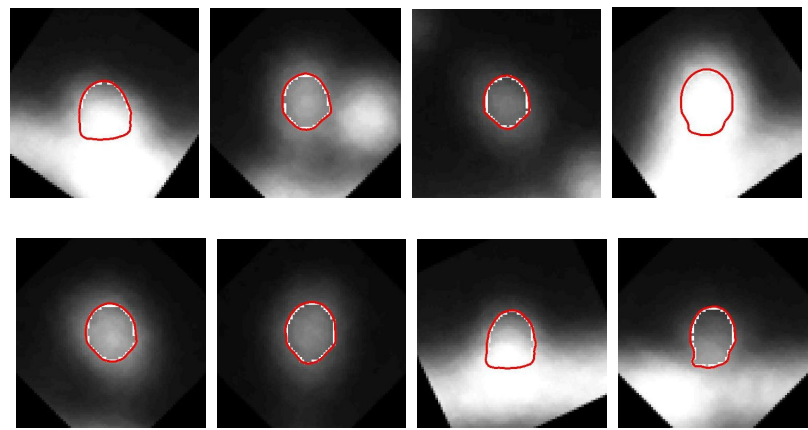

Fig. 4: Visual segmentation results obtained by the approach of Kim et al. [10].

can be achieved by characterizing the posterior distribution in the first time point using a shape sampling approach [16].

\section{ACKNOWLEDGMENT}

This work has been supported by the Scientific and Technological Research Council of Turkey (TUBITAK) under Grant $113 \mathrm{E} 603$.

\section{REFERENCES}

[1] K. M. Harris, "Structure, development, and plasticity of dendritic spines," Current opinion in neurobiology, vol. 9, no. 3, pp. 343-348, 1999.

[2] J. Lippman and A. Dunaevsky, "Dendritic spine morphogenesis and plasticity," Journal of neurobiology, vol. 64, no. 1, pp. 47-57, 2005.

[3] M. Fischer, S. Kaech, D. Knutti, and A. Matus, "Rapid actin-based plasticity in dendritic spines," Neuron, vol. 20, no. 5, pp. 847-854, 1998.

[4] S. Knafo, L. Alonso-Nanclares, J. Gonzalez-Soriano, P. Merino-Serrais, I. Fernaud-Espinosa, I. Ferrer, and J. DeFelipe, "Widespread changes in dendritic spines in a model of alzheimer's disease," Cerebral Cortex, vol. 19, no. 3, pp. 586-592, 2009.

[5] M. Matsuzaki, N. Honkura, G. C. Ellis-Davies, and H. Kasai, "Structural basis of long-term potentiation in single dendritic spines," Nature, vol. 429, no. 6993, pp. 761-766, 2004.

[6] A. Govindarajan, I. Israely, S.-Y. Huang, and S. Tonegawa, "The dendritic branch is the preferred integrative unit for protein synthesisdependent ltp," Neuron, vol. 69, no. 1, pp. 132-146, 2011.

[7] W. Bai, X. Zhou, L. Ji, J. Cheng, and S. T. C. Wong, "Automatic dendritic spine analysis in two-photon laser scanning microscopy images," Cytometry Part A, vol. 71, no. 10, pp. 818-826, 2007.

[8] J. Cheng, X. Zhou, E. Miller, R. M. Witt, J. Zhu, B. L. Sabatini, and S. T. C. Wong, "A novel computational approach for automatic dendrite spines detection in two-photon laser scan microscopy," Journal of neuroscience methods, vol. 165, no. 1, pp. 122-134, 2007.

[9] A. Tsai, A. Yezzi, W. Wells, C. Tempany, D. Tucker, A. Fan, W. E. Grimson, and A. Willsky, "A shape-based approach to the segmentation of medical imagery using level sets," IEEE transactions on medical imaging, vol. 22, no. 2, pp. 137-154, 2003.

[10] J. Kim, M. Çetin, and A. S. Willsky, "Nonparametric shape priors for active contour-based image segmentation," Signal Processing, vol. 87, no. 12, pp. 3021-3044, 2007.

[11] E. Erdil, A. O. Argunsah, T. Tasdizen, D. Unay, and M. Cetin, "A joint classification and segmentation approach for dendritic spine segmentation in 2-photon microscopy images," in Biomedical Imaging (ISBI), 2015 IEEE 12th International Symposium on. IEEE, 2015, pp. 797800.

[12] E. Erdil, L. Rada, A. O. Argunsah, I. Israely, D. Unay, T. Tasdizen, and M. Cetin, "Nonparametric joint shape and feature priors for segmentation of dendritic spines," in Biomedical Imaging (ISBI), 2016 IEEE 13th International Symposium on. IEEE, 2016, pp. 343-346.

[13] T. F. Chan and L. A. Vese, "Active contours without edges," IEEE Transactions on image processing, vol. 10, no. 2, pp. 266-277, 2001.
[14] D. Mumford and J. Shah, "Optimal approximations by piecewise smooth functions and associated variational problems," Communications on pure and applied mathematics, vol. 42, no. 5, pp. 577-685, 1989.

[15] L. R. Dice, "Measures of the amount of ecologic association between species," Ecology, vol. 26, no. 3, pp. 297-302, 1945.

[16] E. Erdil, S. Yildirim, M. Cetin, and T. Tasdizen, "Mcmc shape sampling for image segmentation with nonparametric shape priors," in Proceedings of the IEEE Conference on Computer Vision and Pattern Recognition, 2016, pp. 411-419. 